\title{
SYMMETRIES OF THE BLACK-SCHOLES EQUATION*
}

PAUL LESCOT ${ }^{\dagger}$

Abstract. We study the algebro-geometrical structure of the Black-Scholes equation via the method of isovectors.

Key words. Black-Scholes, isovectors, Lie algebras.

AMS subject classifications. 34A26, 91B28.

1. Introduction. The Black-Scholes pricing formula (see [Black-Scholes 1973], p. 644) is the keystone of modern financial mathematics. In most textbooks (see e.g. [Lamberton-Lapeyre 2012], pp. 88-91), its derivation proceeds via a no-arbitrage argument.

Black and Scholes' original procedure ([Black-Scholes 1973], pp. 642-644) was however quite different : they established a partial differential equation satisfied by the call option price, which they then solved using a reduction to the heat equation (valid under the implicit assumption that, with the notations below, $\tilde{r} \neq 0$ ). To that end, they introduced an apparently completely artificial change of variables and of unknown function ([Black-Scholes 1973], p. 643).

The computation of the symmetry group of a partial differential equation using the method of isovectors ([Harrison-Estabrook 1971]) has already led, in combination with stochastic analysis, to new results in Euclidean Quantum Mechanics ([Lescot-Zambrini 2004], [Lescot-Zambrini 2008]) as well as in ordinary Quantum Mechanics ([Albeverio-Rezende-Zambrini 2006]). More recently, the same circle of ideas has been used in Mathematical Finance ([Lescot 2011], [Quintard 2011]). It was therefore natural to try and apply directly (that is, without a preliminary reduction to the heat equation) the method in question to the Black-Scholes partial differential equation.

After setting the general framework ( $\S 2)$, and performing some preliminary reductions $(\S 3)$, we determine $(\S 4)$ the isovectors for the Black-Scholes equation in a way broadly similar to the one used for the backward heat equation with potential term in the second of the two aforementioned joint papers with J.-C. Zambrini. Our computation turns out to suggest Black and Scholes' original solution method of their equation; in particular, the quantities $r-\frac{\sigma^{2}}{2}$ and $r+\frac{\sigma^{2}}{2}$ appear naturally in this context. As corollaries, we determine ( $\$ 5)$ the structure of the Lie algebra of the symmetry group of the equation, then $(\S 6)$ we obtain some interesting transformations on the solutions.

2. Generalities and notations. We shall be concerned with the classical BlackScholes equation :

$$
\frac{\partial C}{\partial t}+\frac{1}{2} \sigma^{2} S^{2} \frac{\partial^{2} C}{\partial S^{2}}+r S \frac{\partial C}{\partial S}-r C=0(\mathcal{E})
$$

\footnotetext{
*Received April 12, 2011; accepted for publication October 18, 2012

†Laboratoire de Mathématiques Raphaël Salem, UMR 6085 CNRS, Université de Rouen, Technopôle du Madrillet, Avenue de l'Université, B.P. 12, 76801 Saint-Etienne-du-Rouvray, France (Paul.Lescot@univ-rouen.fr).
} 
for the price $C(t, S)$ of a call option with maturity $T$ and strike price $K$ on an underlying asset satisfying $S_{t}=S$ (see [Black-Scholes 1973], where $\sigma$ is denoted by $v, C$ by $w$, and $S$ by $x$ ). As is well-known ([Black-Scholes 1973], p.646), the same equation is satisfied by the price of a put option.

We assume $\sigma>0$, and define

$$
\tilde{r}:=r-\frac{\sigma^{2}}{2}
$$

and

$$
\tilde{s}:=r+\frac{\sigma^{2}}{2}
$$

It is useful to remark that

$$
\frac{\tilde{r}^{2}}{2 \sigma^{2}}+r=\frac{\tilde{s}^{2}}{2 \sigma^{2}}
$$

We intend to determine the isovectors for $(\mathcal{E})$, using the method applied, in [Harrison-Estabrook 1971], pp. 657-658 (see also [Lescot-Zambrini 2004], pp. 189192 ) to the heat equation, and in [Lescot-Zambrini 2008], §3, to the (backward) heat equation with a potential term.

Let us set $x=\ln (S)$ and

$$
\varphi(t, x):=C\left(t, e^{x}\right)=C(t, S) ;
$$

then $\varphi$ is defined on $\mathbf{R}_{+} \times \mathbf{R}$. One has

$$
\begin{gathered}
\frac{\partial C}{\partial S}=\frac{1}{S} \frac{\partial \varphi}{\partial x} \\
\frac{\partial^{2} C}{\partial S^{2}}=-\frac{1}{S^{2}} \frac{\partial \varphi}{\partial x}+\frac{1}{S^{2}} \frac{\partial^{2} \varphi}{\partial x^{2}}
\end{gathered}
$$

and

$$
\frac{\partial C}{\partial t}=\frac{\partial \varphi}{\partial t}
$$

Equation $(\mathcal{E})$ is therefore equivalent to the following equation in $\varphi$ :

$$
\frac{\partial \varphi}{\partial t}+\frac{\sigma^{2}}{2}\left(\frac{\partial^{2} \varphi}{\partial x^{2}}-\frac{\partial \varphi}{\partial x}\right)+r \frac{\partial \varphi}{\partial x}-r \varphi=0\left(\mathcal{E}_{1}\right)
$$

that is :

$$
\frac{\partial \varphi}{\partial t}+\frac{\sigma^{2}}{2} \frac{\partial^{2} \varphi}{\partial x^{2}}+\tilde{r} \frac{\partial \varphi}{\partial x}-r \varphi=0\left(\mathcal{E}_{2}\right)
$$

3. Computation of the isovectors: preliminary reductions. Let us set $A=\frac{\partial \varphi}{\partial x}$ and $B=\frac{\partial \varphi}{\partial t}$, and consider thenceforth $t, x, \varphi, A$ and $B$ as independent variables . Then $\left(\mathcal{E}_{2}\right)$ is equivalent to the vanishing, on the five-dimensional manifold $M=\mathbf{R}_{+} \times \mathbf{R}^{4}$ of $(t, x, \varphi, A, B)$, of the following system of differential forms :

$$
\alpha=d \varphi-A d x-B d t,
$$




$$
d \alpha=-d A d x-d B d t
$$

and

$$
\beta=(B+\tilde{r} A-r \varphi) d x d t+\frac{1}{2} \sigma^{2} d A d t .
$$

Let $I$ denote the ideal of $\Lambda T^{*}(M)$ generated by $\alpha, d \alpha$ and $\beta$; as

$$
\begin{aligned}
d \beta & =(d B+\tilde{r} d A-r d \varphi) d x d t \\
& =d \alpha(d x-\tilde{r} d t)+\alpha(-r d x d t) \in I,
\end{aligned}
$$

$I$ is a differential ideal of $\Lambda T^{*}(M)$. By definition (see [Harrison-Estabrook 1971]), an isovector for $\left(\mathcal{E}_{2}\right)$ is a vector field

$$
N=N^{t} \frac{\partial}{\partial t}+N^{x} \frac{\partial}{\partial x}+N^{\varphi} \frac{\partial}{\partial \varphi}+N^{A} \frac{\partial}{\partial A}+N^{B} \frac{\partial}{\partial B}
$$

such that

$$
\mathcal{L}_{N}(I) \subseteq I .
$$

Using the formal properties of the Lie derivative ([Harrison-Estabrook 1971], p.654), one easily proves that the set $\mathcal{G}$ of these isovectors constitutes a Lie algebra (for the usual bracket of vector fields).

In order to determine $\mathcal{G}$, we may use a trick first explained in [Harrison-Estabrook 1971], p. 657, that applies in all situations in which there is only one 1-form among the given generators of the ideal $I$ (see also [Lescot-Zambrini 2008], p. 211).

Let $N \in \mathcal{G} ;$ as $\mathcal{L}_{N}(I) \subseteq I$, one has $\mathcal{L}_{N}(\alpha) \in I=<\alpha, d \alpha, \beta>$, whence there is a 0 -form (i.e. a function) $\lambda$ such that $\mathcal{L}_{N}(\alpha)=\lambda \alpha$. Let us define

$$
F:=N\rfloor \alpha=N^{\varphi}-A N^{x}-B N^{t} .
$$

This can be rewritten as

$$
\left.\left.\left.\lambda \alpha=\mathcal{L}_{N}(\alpha)=N\right\rfloor d \alpha+d(N\rfloor \alpha\right)=N\right\rfloor d \alpha+d F,
$$

whence

$$
N\rfloor d \alpha=\lambda \alpha-d F,
$$

that is

$$
N\rfloor(-d A d x-d B d t)=\lambda \alpha-d F
$$

i.e.

$$
\begin{aligned}
-N^{A} d x+N^{x} d A-N^{B} d t+N^{t} d B & =\lambda \alpha-d F \\
& =\lambda(d \varphi-A d x-B d t)-d F .
\end{aligned}
$$


Whence (letters as lower indices indicating differentiation, as usual)

$$
\left\{\begin{array}{l}
-N^{B}=-\lambda B-F_{t} \\
-N^{A}=-\lambda A-F_{x} \\
0=\lambda-F_{\varphi} \\
N^{x}=-F_{A} \\
N^{t}=-F_{B} .
\end{array}\right.
$$

Using the third equation, we can eliminate $\lambda$ and obtain

$$
\left\{\begin{array}{l}
N^{t}=-F_{B} \\
N^{x}=-F_{A} \\
N^{\varphi}=F-A F_{A}-B F_{B} \\
N^{A}=F_{x}+A F_{\varphi} \\
N^{B}=F_{t}+B F_{\varphi} .
\end{array}\right.
$$

Conversely, the existence of a function $F(t, x, \varphi, A, B)$ such that the above equations hold clearly implies that $\mathcal{L}_{N}(\alpha)=F_{\varphi} \alpha \in I$; but then

$$
\mathcal{L}_{N}(d \alpha)=d\left(\mathcal{L}_{N}(\alpha)\right) \in d(I) \subseteq I,
$$

and there only remains to be satisfied the condition

$$
\mathcal{L}_{N}(\beta) \in I .
$$

4. The general isovector. The last condition in the previous paragraph can be stated as

$$
\mathcal{L}_{N}(\beta)=\rho \alpha+\xi d \alpha+\omega \beta,
$$

for $\rho$ a 1 -form, $\xi$ a 0 -form and $\omega$ a 0 -form. Let $D$ denote the coefficient of $d \varphi$ in $\rho$; replacing $\rho$ by $\rho-D \alpha$ (which doesn't affect the validity of (4.1) as $\alpha^{2}=0$ ), we may assume that $D=0$. Setting then

$$
\begin{gathered}
\rho=R_{1} d t+R_{2} d x+R_{3} d A+R_{4} d B, \\
\xi=R_{5},
\end{gathered}
$$

and

$$
\omega=R_{6},
$$

we shall obtain a system of ten equations in $F$; we shall then eliminate $R_{1}, \ldots, R_{6}$.

Identifying the coefficients of, in that order, $d t d x, d t d \varphi, d t d A, d t d B, d x d \varphi, d x d A$, $d x d B, d \varphi d A, d \varphi d B$ and $d A d B$ yields the following system :

$$
\begin{gathered}
r N^{\varphi}-\tilde{r} N^{A}-N^{B}+(r \varphi-\tilde{r} A-B) N_{x}^{x}+(r \varphi-\tilde{r} A-B) N_{t}^{t}-\frac{1}{2} \sigma^{2} N_{x}^{A} \\
=-A R_{1}+B R_{2}-(B+\tilde{r} A-r \varphi) R_{6} \\
(r \varphi-\tilde{r} A-B) N_{\varphi}^{x}-\frac{1}{2} \sigma^{2} N_{\varphi}^{A}=R_{1}
\end{gathered}
$$




$$
\begin{gathered}
(r \varphi-\tilde{r} A-B) N_{A}^{x}-\frac{1}{2} \sigma^{2} N_{A}^{A}-\frac{1}{2} \sigma^{2} N_{t}^{t}=B R_{3}-\frac{1}{2} \sigma^{2} R_{6} \\
(r \varphi-\tilde{r} A-B) N_{B}^{x}-\frac{1}{2} \sigma^{2} N_{B}^{A}=B R_{4}+R_{5} \\
(B+\tilde{r} A-r \varphi) N_{\varphi}^{t}=R_{2} \\
(B+\tilde{r} A-r \varphi) N_{A}^{t}-\frac{1}{2} \sigma^{2} N_{x}^{t}=A R_{3}+R_{5} \\
(B+\tilde{r} A-r \varphi) N_{B}^{t}=A R_{4} \\
-\frac{1}{2} \sigma^{2} N_{\varphi}^{t}=-R_{3} \\
0=-R_{4} \\
\frac{1}{2} \sigma^{2} N_{B}^{t}=0 .
\end{gathered}
$$

Equation (4.13) gives $R_{4}=0$; then (4.8) defines $R_{5}$. Equation (4.14) is equivalent to $N_{B}^{t}=0$; if that be the case, then (4.11) holds automatically. Now (4.9) defines $R_{2}$, (4.12) defines $R_{3}$, (4.6) defines $R_{1}$ and (4.7) defines $R_{6}$. We are left with equations (4.5) and (4.10) and the condition $N_{B}^{t}=0$.

Let us begin with the last mentioned ; it is equivalent to $F_{B B}=0: F$ is affine in $B$, i.e. $F=c+B d$, where $c$ and $d$ depend only on $(t, x, \varphi, A)$. Now (3.12) can be rewritten as

$$
\left\{\begin{array}{l}
N^{t}=-d \\
N^{x}=-c_{A}-B d_{A} \\
N^{\varphi}=c-A c_{A}-A B d_{A} \\
N^{A}=c_{x}+B d_{x}+A c_{\varphi}+A B d_{\varphi} \\
N^{B}=c_{t}+B d_{t}+B c_{\varphi}+B^{2} d_{\varphi}
\end{array}\right.
$$

From (4.12) follows

$$
R_{3}=-\frac{1}{2} \sigma^{2} d_{\varphi},
$$

and (4.8) yields

$$
R_{5}=(B+\tilde{r} A-r \varphi) d_{A}-\frac{1}{2} \sigma^{2}\left(d_{x}+A d_{\varphi}\right) .
$$


Now we can rewrite (4.10) as

$$
\begin{aligned}
& (B+\tilde{r} A-r \varphi)\left(-d_{A}\right)-\frac{1}{2} \sigma^{2}\left(-d_{x}\right) \\
= & (B+\tilde{r} A-r \varphi) d_{A}-\frac{1}{2} \sigma^{2}\left(d_{x}+A d_{\varphi}\right)-\frac{1}{2} \sigma^{2} A d_{\varphi} .
\end{aligned}
$$

Comparing the coefficients of $B$ on both sides gives $-d_{A}=d_{A}$, that is $d_{A}=0$, i.e. $d$ depends only upon $(t, x, \varphi)$. Now (4.17) becomes

$$
\frac{1}{2} \sigma^{2} d_{x}=-\frac{1}{2} \sigma^{2}\left(d_{x}+A d_{\varphi}\right)-\frac{1}{2} \sigma^{2} A d_{\varphi}
$$

that is

$$
d_{x}=-A d_{\varphi}
$$

Differentiating with respect to $A$ leads to

$$
0=d_{A x}=-d_{\varphi}
$$

whence $d_{\varphi}=0$ and $d_{x}=-A d_{\varphi}=0: d$ depends only upon $t$. Then (4.15) becomes

$$
\left\{\begin{array}{l}
N^{t}=-d \\
N^{x}=-c_{A} \\
N^{\varphi}=c-A c_{A} \\
N^{A}=c_{x}+A c_{\varphi} \\
N^{B}=c_{t}+B d_{t}+B c_{\varphi}
\end{array}\right.
$$

the new unknowns being a function $c(t, x, \varphi, A)$ and a function $d(t)$, and we still have to satisfy equation (4.5). Now (4.9) implies $R_{2}=0$, and (4.6) gives

$$
R_{1}=-(r \varphi-\tilde{r} A-B) c_{A \varphi}-\frac{1}{2} \sigma^{2}\left(c_{x \varphi}+A c_{\varphi \varphi}\right)
$$

Equation (4.7) now becomes

$$
(r \varphi-\tilde{r} A-B)\left(-c_{A A}\right)-\frac{1}{2} \sigma^{2}\left(c_{x A}+c_{\varphi}+A c_{A \varphi}\right)-\frac{1}{2} \sigma^{2}\left(-d_{t}\right)=-\frac{1}{2} \sigma^{2} R_{6},
$$

that is :

$$
R_{6}=-d_{t}+c_{x A}+c_{\varphi}+A c_{A \varphi}+\frac{2}{\sigma^{2}}(B+\tilde{r} A-r \varphi)\left(-c_{A A}\right)
$$

Eliminating $R_{1}, R_{2}$ and $R_{6}$ turns (4.5) into

$$
\begin{aligned}
& r\left(c-A c_{A}\right)-\tilde{r}\left(c_{x}+A c_{\varphi}\right)-\left(c_{t}+B d_{t}+B c_{\varphi}\right)+(r \varphi-\tilde{r} A-B)\left(-c_{A x}\right) \\
+ & (r \varphi-\tilde{r} A-B)\left(-d_{t}\right)-\frac{1}{2} \sigma^{2}\left(c_{x x}+A c_{\varphi x}\right) \\
= & -A\left(-(r \varphi-\tilde{r} A-B) c_{A, \varphi}-\frac{1}{2} \sigma^{2}\left(c_{x \varphi}+A c_{\varphi \varphi}\right)\right) \\
(4.26) \quad & -(B+\tilde{r} A-r \varphi)\left(-d_{t}+c_{x A}+c_{\varphi}+A c_{A \varphi}+\frac{2}{\sigma^{2}}(B+\tilde{r} A-r \varphi)\left(-c_{A A}\right)\right) .
\end{aligned}
$$


Both members of (4.26) are second-order polynomials in $B$; equating the coefficients of $B^{2}$ gives $c_{A A}=0$, whence $c$ is affine in $A: c=e+A f$ with $e$ and $f$ functions of $(t, x, \varphi)$.

Equating the coefficients of $B$ yields

$$
-d_{t}-c_{\varphi}+c_{A x}+d_{t}=-A c_{A \varphi}-\left(-d_{t}+c_{A x}+c_{\varphi}+A c_{A \varphi}\right)
$$

that is :

$$
d_{t}=2 c_{A x}+2 A c_{A \varphi}=2 f_{x}+2 A f_{\varphi} .
$$

Differentiating the last equality with respect to $A$ gives $f_{\varphi}=0$ (that is, $f$ is a function of $(t, x))$, and then we get that $d_{t}=2 f_{x}$, i.e.

$$
f=\frac{1}{2} d^{\prime}(t) x+\mu(t)
$$

for some function $\mu$ of $t$ alone.

Equating the constant terms in $B$ now gives us

$$
\begin{aligned}
& r e-\tilde{r}\left(e_{x}+A f_{x}+A e_{\varphi}\right)-\left(e_{t}+A f_{t}\right)+(r \varphi-\tilde{r} A)\left(-f_{x}\right) \\
& +(r \varphi-\tilde{r} A)\left(-d_{t}\right)-\frac{1}{2} \sigma^{2}\left(e_{x x}+A f_{x x}+A e_{\varphi x}\right) \\
= & A \frac{1}{2} \sigma^{2}\left(e_{x \varphi}+A e_{\varphi \varphi}\right)-(\tilde{r} A-r \varphi)\left(-d_{t}+f_{x}+e_{\varphi}\right) .
\end{aligned}
$$

Both sides of equation (4.30) are polynomials in $A$ with coefficients depending only upon $(t, x, \varphi)$. Identifying the terms in $A^{2}$ leads us to $e_{\varphi \varphi}=0$, whence $e=g+h \varphi$ with $g$ and $h$ depending only upon $(t, x)$. The unknowns are now $d$ and $\mu$ (functions of $t$ alone) and $g$ and $h$ (functions of $(t, x)$ ).

Identifying the coefficients of $A$ on both sides of (4.30) yields

$$
\begin{aligned}
& -\tilde{r}\left(f_{x}+e_{\varphi}\right)-f_{t}+\tilde{r} f_{x}+\tilde{r} d_{t}-\frac{1}{2} \sigma^{2}\left(f_{x x}+e_{\varphi x}\right) \\
= & \frac{1}{2} \sigma^{2} e_{x \varphi}-\tilde{r}\left(-d_{t}+f_{x}+e_{\varphi}\right),
\end{aligned}
$$

that is

$$
-f_{t}-\frac{1}{2} \sigma^{2} f_{x x}=\sigma^{2} h_{x}-\tilde{r} f_{x}
$$

or

$$
\sigma^{2} h_{x}=\tilde{r} \frac{1}{2} d^{\prime}(t)-\frac{1}{2} d^{\prime \prime}(t) x-\mu^{\prime}(t)
$$

that is

$$
h(t, x)=\frac{\tilde{r}}{2 \sigma^{2}} d^{\prime}(t) x-\frac{d^{\prime \prime}(t)}{4 \sigma^{2}} x^{2}-\frac{\mu^{\prime}(t)}{\sigma^{2}} x+k(t),
$$

for some function $k$ of $t$ alone. 
The constant term gives

$$
r e-\tilde{r} e_{x}-e_{t}-r \varphi f_{x}-r \varphi d_{t}-\frac{1}{2} \sigma^{2} e_{x x}=r \varphi\left(-d_{t}+f_{x}+e_{\varphi}\right) .
$$

According to the relation $d_{t}=2 f_{x}$, this becomes

$$
r g-\tilde{r} g_{x}-\tilde{r} \varphi h_{x}-g_{t}-\varphi h_{t}-r \varphi d_{t}-\frac{1}{2} \sigma^{2} g_{x x}-\frac{1}{2} \sigma^{2} \varphi h_{x x}=0 .
$$
$\left(\mathcal{E}_{2}\right)$.

Equating the constant term (in $\varphi$ ) of (4.36) to zero gives that $g$ is a solution of

The value of the term in $\varphi$ means that

$$
-\tilde{r} h_{x}-h_{t}-r d_{t}-\frac{1}{2} \sigma^{2} h_{x x}=0
$$

that is

$$
\begin{aligned}
& -\tilde{r}\left(\frac{\tilde{r}}{2 \sigma^{2}} d^{\prime}(t)-\frac{d^{\prime \prime}(t)}{2 \sigma^{2}} x-\frac{\mu^{\prime}(t)}{\sigma^{2}}\right) \\
& -\left(\frac{\tilde{r}}{2 \sigma^{2}} d^{\prime \prime}(t) x-\frac{d^{\prime \prime \prime}(t)}{4 \sigma^{2}} x^{2}-\frac{\mu^{\prime \prime}(t)}{\sigma^{2}} x+k^{\prime}(t)\right)-r d^{\prime}(t)-\frac{1}{2} \sigma^{2}\left(-\frac{d^{\prime \prime}(t)}{2 \sigma^{2}}\right)=0 .
\end{aligned}
$$

This is a polynomial equation in $x$ with functions of $t$ as coefficients. Considering the coefficient of $x^{2}$ gives $d^{\prime \prime \prime}(t)=0$, that is $d(t)=C_{1} t^{2}+C_{2} t+C_{3}$ for constants $C_{1}$, $C_{2}$ and $C_{3}$.

Equating the terms in $x$ leads to

$$
\frac{\tilde{r}}{2 \sigma^{2}} d^{\prime \prime}(t)-\frac{\tilde{r}}{2 \sigma^{2}} d^{\prime \prime}(t)+\frac{\mu^{\prime \prime}(t)}{\sigma^{2}}=0
$$

that is

$$
\mu^{\prime \prime}(t)=0
$$

or

$$
\mu(t)=C_{4} t+C_{5}
$$

$\left(C_{4}, C_{5}\right.$ real constants).

We are left with the constant term in $x$ :

$$
-\frac{\tilde{r}^{2}}{2 \sigma^{2}} d^{\prime}(t)+\tilde{r} \frac{\mu^{\prime}(t)}{\sigma^{2}}-k^{\prime}(t)-r d^{\prime}(t)+\frac{d^{\prime \prime}(t)}{4}=0,
$$

or

$$
\begin{aligned}
k(t) & =-\left(\frac{\tilde{r}^{2}}{2 \sigma^{2}}+r\right) d(t)+\tilde{r} \frac{\mu(t)}{\sigma^{2}}+\frac{d^{\prime}(t)}{4}+C_{6} \\
& =-\frac{\tilde{s}^{2}}{2 \sigma^{2}} d(t)+\tilde{r} \frac{\mu(t)}{\sigma^{2}}+\frac{d^{\prime}(t)}{4}+C_{6}
\end{aligned}
$$

for some constant $C_{6}$. Therefore

$$
\begin{aligned}
f(t, x) & =\frac{1}{2} d^{\prime}(t) x+\mu(t) \\
& =\frac{1}{2} x\left(2 C_{1} t+C_{2}\right)+C_{4} t+C_{5}
\end{aligned}
$$


and

$$
\begin{aligned}
h(t, x) & =\frac{\tilde{r}}{2 \sigma^{2}} x\left(2 C_{1} t+C_{2}\right)-\frac{C_{1}}{2 \sigma^{2}} x^{2} \\
& -\frac{C_{4} x}{\sigma^{2}}-\frac{\tilde{s}^{2}}{2 \sigma^{2}}\left(C_{1} t^{2}+C_{2} t+C_{3}\right) \\
& +\tilde{r} \frac{\left(C_{4} t+C_{5}\right)}{\sigma^{2}}+\frac{2 C_{1} t+C_{2}}{4}+C_{6} .
\end{aligned}
$$

We have established

TheOREM 4.1. The general isovector $N$ for $\left(\mathcal{E}_{2}\right)$ is given, in terms of an arbitrary solution $g$ of $\left(\mathcal{E}_{2}\right)$ and six arbitrary real constants $C_{1}, \ldots, C_{6}$, by the formulas

$$
\begin{aligned}
& N^{t}=-C_{1} t^{2}-C_{2} t-C_{3} \\
& N^{x}=-\frac{1}{2} x\left(2 C_{1} t+C_{2}\right)-\left(C_{4} t+C_{5}\right) \\
& N^{\varphi}=g+\varphi\left(\frac{\tilde{r}}{2 \sigma^{2}} x\left(2 C_{1} t+C_{2}\right)-\frac{C_{1}}{2 \sigma^{2}} x^{2}-\frac{C_{4} x}{\sigma^{2}}\right. \\
& \left.-\frac{\tilde{s}^{2}}{2 \sigma^{2}}\left(C_{1} t^{2}+C_{2} t+C_{3}\right)+\tilde{r} \frac{C_{4} t+C_{5}}{\sigma^{2}}+\frac{2 C_{1} t+C_{2}}{4}+C_{6}\right) \\
& N^{A}=g_{x}+\varphi\left(\frac{\tilde{r}}{2 \sigma^{2}}\left(2 C_{1} t+C_{2}\right)-\frac{C_{1} x}{\sigma^{2}}-\frac{C_{4}}{\sigma^{2}}\right) \\
& +\frac{A}{2}\left(2 C_{1} t+C_{2}\right) \\
& +A\left(\frac{\tilde{r}}{2 \sigma^{2}} x\left(2 C_{1} t+C_{2}\right)-\frac{C_{1}}{2 \sigma^{2}} x^{2}\right. \\
& -\frac{C_{4} x}{\sigma^{2}}-\frac{\tilde{s}^{2}}{2 \sigma^{2}}\left(C_{1} t^{2}+C_{2} t+C_{3}\right) \\
& \left.+\tilde{r} \frac{\left(C_{4} t+C_{5}\right)}{\sigma^{2}}+\frac{2 C_{1} t+C_{2}}{4}+C_{6}\right) \text {. } \\
& N^{B}=g_{t}+\varphi\left(\frac{\tilde{r} x}{\sigma^{2}}-\frac{\tilde{s}^{2}}{2 \sigma^{2}}\left(2 C_{1} t+C_{2}\right)+\frac{\tilde{r} C_{4}}{\sigma^{2}}+\frac{C_{1}}{2}\right) \\
& +A\left(x C_{1}+C_{4}\right)+B\left(2 C_{1} t+C_{2}\right) \\
& +B\left(\frac{\tilde{r}}{2 \sigma^{2}} x\left(2 C_{1} t+C_{2}\right)-\frac{C_{1}}{2 \sigma^{2}} x^{2}\right. \\
& -\frac{C_{4} x}{\sigma^{2}}-\frac{\tilde{s}^{2}}{2 \sigma^{2}}\left(C_{1} t^{2}+C_{2} t+C_{3}\right) \\
& \left.+\tilde{r} \frac{\left(C_{4} t+C_{5}\right)}{\sigma^{2}}+\frac{2 C_{1} t+C_{2}}{4}+C_{6}\right) \text {. }
\end{aligned}
$$


5. The Lie algebra. For $u$ a solution of $\left(\mathcal{E}_{2}\right)$, let $N_{u}$ denote the isovector defined by $g=u$ and $C_{1}=\ldots=C_{6}=0$, and, for $1 \leq i \leq 6$, let $N_{i}$ denote the isovector defoned by by $g=0, C_{i}=1$ and $C_{j}=0$ for $j \neq i$.

The function $g$ and $h$ are determined by the isovector $N$ :

$$
g=N^{\varphi}-\varphi \frac{\partial N^{\varphi}}{\partial \varphi}
$$

and

$$
h=\frac{\partial N^{\varphi}}{\partial \varphi}
$$

we shall denote them repectively by $g_{N}$ and $h_{N}$. As seen in $\S 4, h_{N}$ is a function of $(t, x)$.

Lemma 5.1. For all $(M, N) \in \mathcal{G}^{2}$,

$$
g_{[M, N]}=M^{t} \frac{\partial g_{N}}{\partial t}+M^{x} \frac{\partial g_{N}}{\partial x}+g_{M} h_{N}-N^{t} \frac{\partial g_{M}}{\partial t}-N^{x} \frac{\partial g_{M}}{\partial x}-g_{N} h_{M}
$$

and

$$
h_{[M, N]}=M^{t} \frac{\partial h_{N}}{\partial t}+M^{x} \frac{\partial h_{N}}{\partial x}-N^{t} \frac{\partial h_{M}}{\partial t}-N^{x} \frac{\partial h_{M}}{\partial x} .
$$

Proof. One has

$$
\begin{aligned}
{[M, N]^{\varphi}=} & M\left(N^{\varphi}\right)-N\left(M^{\varphi}\right) \\
= & M\left(g_{N}+\varphi h_{N}\right)-N\left(g_{M}+\varphi h_{M}\right) \\
= & M^{t} \frac{\partial g_{N}}{\partial t}+M^{x} \frac{\partial g_{N}}{\partial x}+\varphi\left(M^{t} \frac{\partial h_{N}}{\partial t}+M^{x} \frac{\partial h_{N}}{\partial x}\right) \\
& +M^{\varphi} h_{N}-\left(N^{t} \frac{\partial g_{M}}{\partial t}+N^{x} \frac{\partial g_{M}}{\partial x}\right. \\
& \left.+\varphi\left(N^{t} \frac{\partial h_{M}}{\partial t}+N^{x} \frac{\partial h_{M}}{\partial x}\right)+N^{\varphi} h_{M}\right) \\
= & M^{t} \frac{\partial g_{N}}{\partial t}+M^{x} \frac{\partial g_{N}}{\partial x}+\varphi\left(M^{t} \frac{\partial h_{N}}{\partial t}+M^{x} \frac{\partial h_{N}}{\partial x}\right) \\
+ & \left(g_{M}+\varphi h_{M}\right) h_{N}-N^{t} \frac{\partial g_{M}}{\partial t}-N^{x} \frac{\partial g_{M}}{\partial x} \\
& -\varphi\left(N^{t} \frac{\partial h_{M}}{\partial t}+N^{x} \frac{\partial h_{M}}{\partial x}\right)-\left(g_{N}+\varphi h_{N}\right) h_{M} \\
= & \left(M^{t} \frac{\partial g_{N}}{\partial t}+M^{x} \frac{\partial g_{N}}{\partial x}+g_{M} h_{N}-N^{t} \frac{\partial g_{M}}{\partial t}-N^{x} \frac{\partial g_{M}}{\partial x}-g_{N} h_{M}\right) \\
+ & \varphi\left(M^{t} \frac{\partial h_{N}}{\partial t}+M^{x} \frac{\partial h_{N}}{\partial x}-N^{t} \frac{\partial h_{M}}{\partial t}-N^{x} \frac{\partial h_{M}}{\partial x}\right) ;
\end{aligned}
$$

the result follows.

Let us set

$$
\mathcal{H}=\left\{N \in \mathcal{G} \mid g_{N}=0\right\}
$$

and

$$
\mathcal{J}=\left\{N \in \mathcal{G} \mid h_{N}=0\right\}
$$


Proposition 5.2. $\mathcal{J}$ is an ideal of $\mathcal{G}$ and $\mathcal{H}$ is a subalgebra of $\mathcal{G}$. Furthermore $\mathcal{G}=\mathcal{H} \oplus \mathcal{J}, \mathcal{H}$ has dimension 6 and admits $\left(N_{1}, \ldots, N_{6}\right)$ as a basis, and $\mathcal{J}=\left\{N_{u} \mid u\right.$ solution of $\left.\left(\mathcal{E}_{2}\right)\right\}$.

Proof. Clearly, $N \in \mathcal{J}$ if and only if $C_{1}=\ldots=C_{6}=0$; in particular, if $N \in \mathcal{J}$ then $N^{t}=N^{x}=0$. Therefore $M \in \mathcal{G}$ and $N \in \mathcal{J}$ imply $h_{[M, N]}=0$, i.e. $[M, N] \in \mathcal{J}$ : $\mathcal{J}$ is an ideal of $\mathcal{G}$.

Furthermore, $g_{M}=g_{N}=0$ imply $g_{[M, N]}=0: \mathcal{H}$ is a subalgebra of $\mathcal{G}$. The last two assertions clearly hold.

6. Some symmetries. Let $N \in \mathcal{G}$, let $\kappa \in \mathbf{R}$, and let $\varphi$ be a solution of $\left(\mathcal{E}_{2}\right)$; then $e^{\kappa N}$ maps $(t, x, \varphi, A, B)$ to $\left(t_{\kappa}, x_{\kappa}, \varphi_{\kappa}, A_{\kappa}, B_{\kappa}\right)$; setting

$$
\varphi_{\kappa}=\psi_{\kappa}\left(t_{\kappa}, x_{\kappa}\right) \text {, }
$$

it follows that $\psi_{\kappa}$ is also a solution of $\left(\mathcal{E}_{2}\right)$. We shall denote

$$
e^{\kappa \tilde{N}}: \varphi \mapsto \psi_{\kappa}
$$

the associated one-parameter group.

LEMMA 6.1. If

$$
N=N^{t} \frac{\partial}{\partial t}+N^{x} \frac{\partial}{\partial x}+N^{\varphi} \frac{\partial}{\partial \varphi}+\ldots \in \mathcal{G}
$$

then

$$
\tilde{N}(\varphi)=-N^{t} \frac{\partial \varphi}{\partial t}-N^{x} \frac{\partial \varphi}{\partial x}+N^{\varphi},
$$

that is

$$
\begin{aligned}
\tilde{N}(\varphi) & =\left(C_{1} t^{2}+C_{2} t+C_{3}\right) \frac{\partial \varphi}{\partial t}+\left(\frac{1}{2} x\left(2 C_{1} t+C_{2}\right)+\left(C_{4} t+C_{5}\right)\right) \frac{\partial \varphi}{\partial x} \\
& +g+\varphi\left(\frac{\tilde{r}}{2 \sigma^{2}} x\left(2 C_{1} t+C_{2}\right)-\frac{C_{1}}{2 \sigma^{2}} x^{2}-\frac{C_{4} x}{\sigma^{2}}-\frac{\tilde{s}^{2}}{2 \sigma^{2}}\left(C_{1} t^{2}+C_{2} t+C_{3}\right)\right. \\
& \left.+\tilde{r} \frac{C_{4} t+C_{5}}{\sigma^{2}}+\frac{2 C_{1} t+C_{2}}{4}+C_{6}\right) .
\end{aligned}
$$

Proof. Let us rewrite (6.1) as

$$
\left(e^{\kappa N}(\varphi)\right)(t, x)=\left(e^{\kappa \tilde{N}}(\varphi)\right)\left(e^{\kappa N}(t), e^{\kappa N}(x)\right) .
$$

Developping at order one in $\kappa$ gives

$$
\begin{aligned}
\varphi+\kappa N^{\varphi} & =\varphi\left(t+\kappa N^{t}, x+\kappa N^{x}\right)+\kappa \tilde{N}(\varphi)+o(\kappa) \\
& =\varphi+\kappa N^{t} \frac{\partial \varphi}{\partial t}+\kappa N^{x} \frac{\partial \varphi}{\partial x}+\kappa \tilde{N}(\varphi)+o(\kappa),
\end{aligned}
$$

whence the result. 
We shall set

$$
C_{N}^{\kappa}(t, S):=\left(e^{\kappa \tilde{N}} \varphi\right)(t, \ln (S))
$$

and

$$
C_{j}^{\kappa}:=C_{N_{j}}^{\kappa}
$$

$(1 \leq j \leq 6)$.

Some of these transforms can actually be explicitly computed; for instance

$$
\tilde{N}_{3}(\varphi)=\frac{\partial \varphi}{\partial t}-\frac{\tilde{s}^{2}}{2 \sigma^{2}} \varphi
$$

whence

$$
\left(e^{\kappa \tilde{N}_{3}} \varphi\right)(t, x)=e^{-\frac{\kappa \tilde{s}^{2}}{2 \sigma^{2}}} \varphi(t+\kappa, x)
$$

and

$$
C_{3}^{\kappa}(t, S)=e^{-\frac{\kappa \tilde{s}^{2}}{2 \sigma^{2}}} C(t+\kappa, S) .
$$

$$
\tilde{N}_{4}(\varphi)=t \frac{\partial \varphi}{\partial x}+\left(\frac{\tilde{r} t-x}{\sigma^{2}}\right) \varphi
$$

therefore

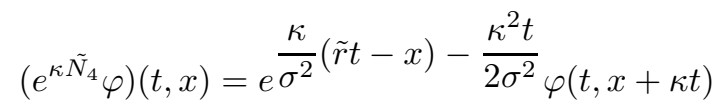

and

$$
C_{4}^{\kappa}(t, S)=e^{\frac{\kappa t}{2 \sigma^{2}}(2 \tilde{r}-\kappa)} S^{-\frac{\kappa}{\sigma^{2}}} C\left(t, e^{\kappa t} S\right) .
$$

$$
\tilde{N}_{5}(\varphi)=\frac{\partial \varphi}{\partial x}+\frac{\tilde{r} \varphi}{\sigma^{2}}
$$

whence

$$
\left(e^{\kappa \tilde{N}_{5}} \varphi\right)(t, x)=e^{\frac{\kappa \tilde{r}}{\sigma^{2}}} \varphi(t, x+\kappa)
$$

and

$$
C_{5}^{\kappa}(t, S)=e^{\frac{\kappa \tilde{r}}{\sigma^{2}}} C\left(t, e^{\kappa} S\right)
$$


Lastly,

$$
\tilde{N}_{6}(\varphi)=\varphi
$$

whence

$$
\left(e^{\kappa \tilde{N}_{6}} \varphi\right)(t, x)=e^{\kappa} \varphi(t, x)
$$

and

$$
C_{6}^{\kappa}(t, S)=e^{\kappa} C(t, S)
$$

Corollary 6.2. Let $C$ denote a solution of $(\mathcal{E})$. Then, for each $\kappa \in \mathbf{R}$, the $C_{i}^{\kappa}(3 \leq i \leq 6)$ defined by $(6.7),(6.10),(6.13)$ and $(6.16)$ are also solutions of $(\mathcal{E})$.

Together the transformations described in (6.7) and (6.16) come from the invariance of the original equation via multiplication of the solution by a scalar and translation in time ; $(6.13)$ then comes from the homogeneity in $S$ - these could be expected. The transformation given by (6.10) is, however, not so easy to understand. It would be interesting to find some financial interpretation for it.

Acknowledgements. I presented prior versions of this work at the Ascona Conference (May 2008), at the seminar of the Grupo de Física-Matemática (Lisbon, July 2008), at the Stochastic Analysis Seminar (Loughborough, January 2010) and at the Séminaire de Sciences Actuarielles (Université Libre de Bruxelles, February 2010). For these invitations I am deeply indebted to (respectively) Professors Robert Dalang, Marco Dozzi and Francesco Russo, Professors Ana Bela Cruzeiro and Jean-Claude Zambrini, Professor József Lörinczi, and Professor Pierre Patie. I am grateful to many members of the various audiences, notably Professors Eckhard Platen and Eric Carlen, for their remarks. I also thank Professor Archil Gulisashvili for his remarks on the original text.

\section{REFERENCES}

[Albeverio-Rezende-Zambrini 2006]

[Black-Scholes 1973]

[Harrison-Estabrook 1971]

[Lamberton-Lapeyre 2012]

[Lescot 2011]

[Lescot-Zambrini 2004]

[Lescot-Zambrini 2008]
S. Albeverio, J. Rezende, and J.-C. Zambrini, Probability and Quantum Symmetries. II. The Theorem of Noether in quantum mechanics, J. Math. Phys., 47 (2006), 062107.

F. Black And M. Scholes, The pricing of options and corporate liabilities, Journal of Political Economy, 81:3 (1973), pp. 637-654.

B. K. Harrison and F. B. Estabrook, Geometric Approach to Invariance Groups and Solution of Partial Differential Systems, J. Math. Phys., 12 (1971), pp. 653-666.

D. LAmberton, B. LAPEyRe, Introduction au Calcul Stochastique Appliqué à la Finance, Ellipses, 2012.

P. LESCOT, On affine interest rate models, 2011. arXiv:0911.2757.

P. LESCOT AND J.-C. ZAMBRINI, Isovectors for the Hamilton-Jacobi-Bellman Equation, Formal Stochastic Differentials and First Integrals in Euclidean Quantum Mechanics, Progress in Probability, vol. 58, Birkhaüser, 2004, pp. 187-202.

P. Lescot AND J.-C. ZAMBRINI, Probabilistic deformation of contact geometry, diffusion processes and their quadratures, Progress in Probability, vol. 59, Birkhaüser, 2008, pp. 203-226. 
[Quintard 2011]

H. Quintard, Processus affines en finance et symétries d'équations aux dérivées partielles, Master's Thesis, Rouen, 2011. 